\title{
Molecular evolution of a-kinase anchoring protein (AKAP)-7: implications in comparative PKA compartmentalization
}

\author{
Keven R Johnson', Jessie Nicodemus-Johnson ${ }^{2}$, Graeme K Carnegie ${ }^{3}$ and Robert S Danziger ${ }^{1,4,5^{*}}$
}

\begin{abstract}
Background: A-Kinase Anchoring Proteins (AKAPs) are molecular scaffolding proteins mediating the assembly of multi-protein complexes containing CAMP-dependent protein kinase A (PKA), directing the kinase in discrete subcellular locations. Splice variants from the AKAP7 gene (AKAP15/18) are vital components of neuronal and cardiac phosphatase complexes, ion channels, cardiac $\mathrm{Ca}^{2+}$ handling and renal water transport.

Results: Shown in evolutionary analyses, the formation of the AKAP7-RI/RIl binding domain (required for AKAP/ PKA-R interaction) corresponds to vertebrate-specific gene duplication events in the PKA-RI/RIl subunits. Species analyses of AKAP7 splice variants shows the ancestral AKAP7 splice variant is AKAP7a, while the ancestral long form AKAP7 splice variant is AKAP7Y. Multi-species AKAP7 gene alignments, show the recent formation of AKAP7 $\delta$ occurs with the loss of native AKAP7Y in rats and basal primates. AKAP7 gene alignments and two dimensional Western analyses indicate that AKAP7Y is produced from an internal translation-start site that is present in the AKAP7 $\delta$ CDNA of mice and humans but absent in rats. Immunofluorescence analysis of AKAP7 protein localization in both rat and mouse heart suggests AKAP7Y replaces AKAP7 $\delta$ at the cardiac sarcoplasmic reticulum in species other than rat. DNA sequencing identified Human AKAP7 $\delta$ insertion-deletions (indels) that promote the production of AKAP7Y instead of AKAP78.

Conclusions: This AKAP7 molecular evolution study shows that these vital scaffolding proteins developed in ancestral vertebrates and that independent mutations in the AKAP7 genes of rodents and early primates has resulted in the recent formation of AKAP7 $\delta$, a splice variant of likely lesser importance in humans than currently described.
\end{abstract}

Keywords: A-Kinase anchoring protein, Protein evolution, PKA regulatory subunit, PKA compartmentalization, AKAP15/18

\section{Background}

cAMP-dependent protein kinase A (PKA) is a broadspecificity serine/threonine protein kinase that is ubiquitously distributed among tissue types, and identified in organisms from fungi to humans. Inside cells, PKA exists as a tetrameric protein composed of two catalytic subunits and a regulatory subunit dimer. The PKA regulatory subunit (PKA-R) functions to maintain kinase inactivity in the absence of cAMP and form proteinprotein interactions that compartmentalize the kinase.

\footnotetext{
* Correspondence: rdanziger@aol.com

${ }^{1}$ Department of Medicine, University of Illinois at Chicago, Chicago, IL, USA

${ }^{4}$ Jesse Brown VA Medical Center, Chicago, IL, USA

Full list of author information is available at the end of the article
}

The selective sequestration or compartmentalization of intracellular PKA has been supported by several studies showing that PKA is concentrated in discrete intracellular locales by A-kinase anchoring proteins, or AKAPs [1-4]. AKAPs bind to the regulatory (i.e., PKA-RI, PKARII or both) subunit dimer of the tetrameric PKA holoenzyme at an antiparallel four-helix domain termed the dimerization and docking (D/D) domain [5-7]. The AKAP-PKA-R interaction functions to sequester PKA in the vicinity of its phosphorylation substrates $[2,8,9]$ and this selective concentration of PKA activity in part, explains how multiple diverse extracellular stimuli can result in highly specific and discrete PKA-mediated phosphorylation events $[10,11]$.

\section{Biomed Central}


Originally named according to their apparent molecular weight, AKAPs are now designated by gene number (i.e., AKAP15/18 splice variants are referred to as AKAP7 splice variants). Currently there are 14 annotated AKAPs (AKAP1 to AKAP14). The PKA-regulatory subunit binding domain of AKAPs is the most recognizable element among the otherwise structurally diverse protein family. AKAPs are classified according to binding specificity for the PKA regulatory subunits; RI-, RII- or both RI and RII PKA regulatory domains (dual specific) [12]. The primary structure (i.e., amino acid sequence) of the PKARI/RIIbinding domain is highly variable among AKAPs, though the secondary structure, which forms an amphipathic helix, contains several conserved hydrophobic residues that determine PKA-R-subunit specificity and binding affinity [13-15]. AKAPs also contain unique localization signal(s) which are responsible for AKAP targeting to sub-cellular compartments, and in many cases, AKAPs also contain enzyme-binding sites that enable the formation of multiprotein signaling complexes [16,17]. Various AKAP targeting domains have been shown to interact with G-protein coupled receptors (GPCRs; [18]), adenylyl cyclases [19], Exchange protein activated by cAMP (EPAC; [20]), and phosphodiesterases (PDEs) [21,22]. Hence, AKAPs function as signal processing hubs specifically linking upstream activation signals to downstream effector proteins.

AKAPs have been shown to regulate neuronal ion channels [23,24], memory and cognition [25-27], reproduction function [22,28], as well as renal [29] and cardiac function [11,30-35]. One AKAP that has been identified in many tissues and a variety of cell signaling pathways is AKAP7 (also known as AKAP15/18). Splice variants of the AKAP7 gene (AKAP7 $\alpha, \beta, \delta$, and $\gamma$ ) are dual-specificity AKAPs that have been extensively studied in central nervous system (CNS), striated muscle ion channel regulation [2,36,37], cardiac calcium cycling [38], and renal aquaporin transport [29].

AKAP7 $\alpha$, the first AKAP7 splice variant identified, is a membrane bound protein containing a lipid modification domain in the amino terminus [39]. AKAP7 $\alpha$ binds to Ltype $\mathrm{Ca}^{2+}$ channels where it regulates the PKA-dependent phosphorylation of the ion channel [40]. AKAP7 $\alpha$ has also been shown to interact with sodium channels in the brain [2], and is required for feedback inhibition of the epithelial $\mathrm{Na}^{+}$channel (ENAC) [41]. AKAP7 $\alpha$-mediated PKA anchoring to ion channels $\left(\mathrm{Ca}^{2+}\right.$ and $\left.\mathrm{Na}^{+}\right)$provides a regulatory mechanism for channel function, whereby anchoring of PKA regulates the phosphorylation state of the ion channel and has a profound effect on channel "open probability" and ion movement $[1,40,42,43]$. Another extensively studied AKAP7 splice variant is AKAP7 $\delta$, which was originally identified in rat kidney [29]. In rat renal principle cells, the APAK7 $\delta$ splice variant functions to anchor PKA to intracellular vesicles containing the aquaporin water channel and is required for aquaporin fusion to the plasma membrane [29]. Also in rats, AKAP7 $\delta$ has been identified in the cardiac sarcoplasmic-endoplasmic reticulum ATPase (SERCA) where it regulates PKA phosphorylation of a SERCA negative regulatory protein, phospholamban (PLN) and controls $\mathrm{Ca}^{2+}$ re-uptake into the sarcoplasmic reticulum (SR) [38]. AKAP7 $\delta$ has become a prime example for the pharmacological potential of PKA-AKAP disruptor molecules, particularly in the treatment of human heart failure $[44,45]$. Much less is known about the AKAP7 $\beta$ and $\gamma$ splice variants. AKAP7 $\beta$ differs from AKAP7 $\alpha$ by the inclusion of a third exon which imparts a unique membrane localization that differs from that found for AKAP7 $\alpha$ [46]. AKAP7 $\gamma$ has been cloned from human pancreas [46] and mouse oocytes [47], though the functional significance of this splice variant remains relatively unknown.

Though appreciation for the importance of AKAPs in cell signaling has grown exponentially in recent years, an analysis of the evolution and development of an AKAP remains absent. This study addresses several key questions in the molecular evolution of AKAP7 proteins including; 1) evolution/genesis of the AKAP7 RI/RII binding domain in relation to the PKA-R D/D domains 2) evolution of the AKAP7 gene structure 3) species analyses of AKAP7 splice variants, 4) sequence determination of previously un-reported mouse and human AKAP7 $\delta$ and 5) evolutionary conservation of key protein regions in short AKAP7 splice variants and rapid change in AKAP7 long form splice variants.

\section{Results}

\section{Evolution of AKAP7 and PKA-R interaction motifs}

The AKAP7 protein domain required for PKA-R binding (AKAP7 RI/RII binding domain; pfam 10470) is detected in transcripts of species from zebrafish to humans (Figure 1). The 3' terminal exon encoding the AKAP7 RI/RII binding domain first appears in the AKAP7 gene of lamprey and is conserved throughout vertebrates, placing AKAP7 binding domain genesis at the base of the vertebrate lineage (Figures 2 and 3). Transcripts containing (encoding) the AKAP7RI/RII binding domain were not identified in Drosophila and Ciona, though transcripts containing high sequence identity to the AKAP-7 NLS were identified (termed AKAP7-like; Figure 1). AKAP7like genes were also identified in Strongylocentrotus purpuratus (Additional file 1: Figure S1), Chlorella variabilis, and Daphnia pulex (not shown). The resultant putative protein products of these AKAP7-like genes lack the AKAP7RI/ RII binding domain and likely the PKA binding functionality of derived AKAPs.

The PKA-R subunit protein domain necessary for AKAP interaction (the Dimerization/Docking, D/D domain; pfam 02197) is conserved in the PKA-R subunits of insects, urochordates, chordates, and vertebrates (with 


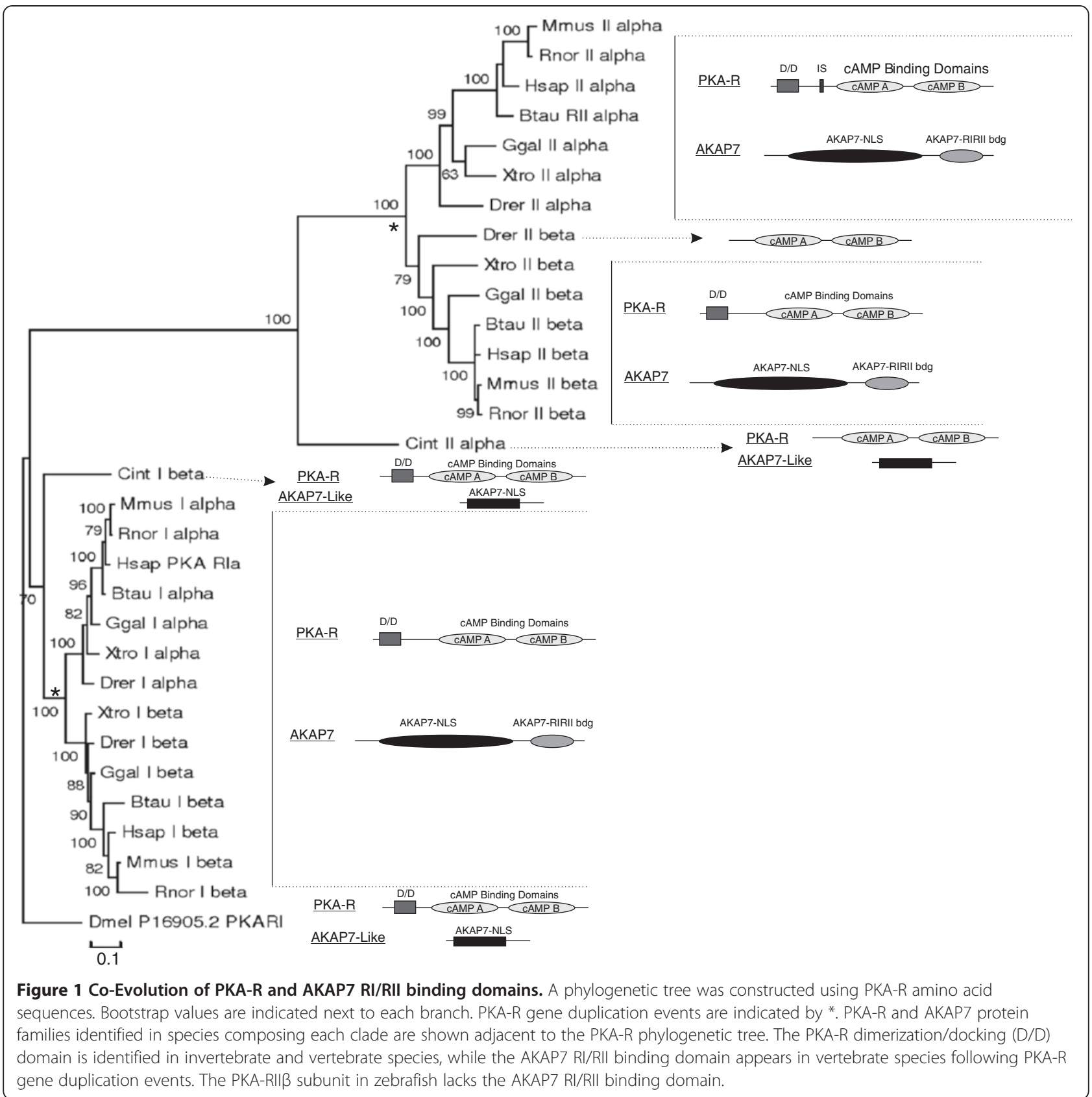

the exception of Zebrafish PKA-RII $\beta$; Figures 1 and 2). Specifically, the residues necessary for PKA-R/AKAP7 interaction $[5,7,13,15]$ are conserved in all species examined (Figure 2). Appearance of the AKAP7 RI/RII binding domain corresponds with the vertebrate specific PKA-R gene duplication (Figure 2).

\section{AKAP7 Splice variant evolution}

AKAP7 gene structure, exon numbers and orientation were determined for multiple species (Figure 3, Additional file 1: Figure S1). AKAP7 $\alpha$ coding sequence in the AKAP7 gene and EST transcript evidence first appears in basal teleosts (Danio rerio), and is conserved from teleosts to humans (Figure 3, Additional file 1: Figure S1). AKAP7 $\beta$ ESTs are only identified in mammals (pig; Sus scrofa; Additional file 1: Figure S1), though the AKAP7 $\beta$-specific gene coding region is detectable from marsupials to mammals (Figure 3). AKAP7 $\delta$ is the most recent AKAP7 splice variant, with EST evidence supporting transcript presence in rats through humans. However exon data suggests this splice variant is absent from AKAP7 genes in species below laurasiatherian mammals (Additional file 1: Figure S1, Figure 4). AKAP7 $\delta$ is formed by the incorporation of a 19-nucleotide exon 

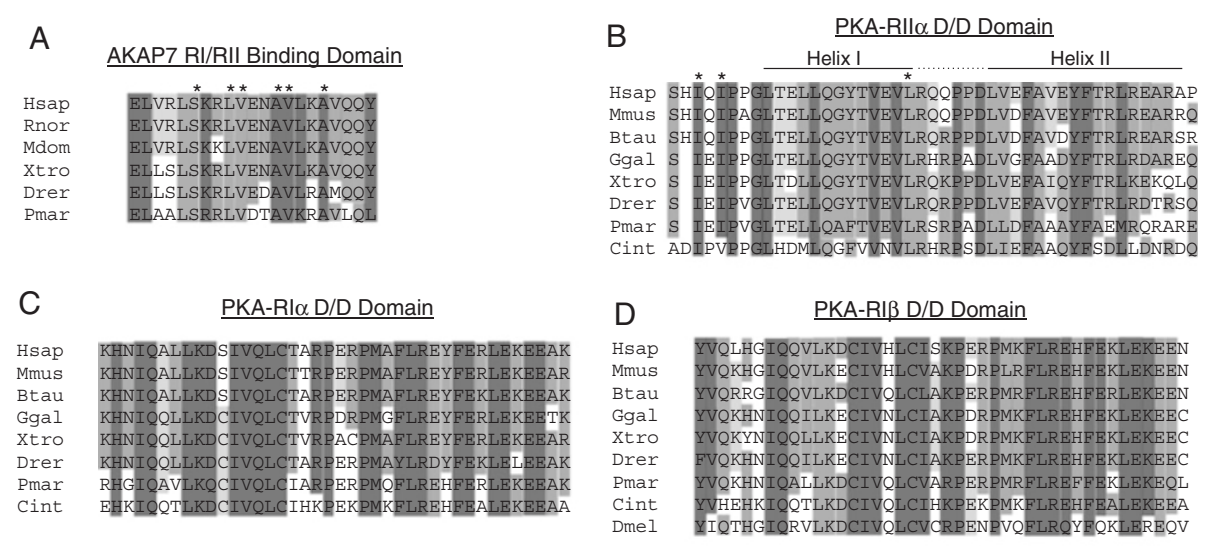

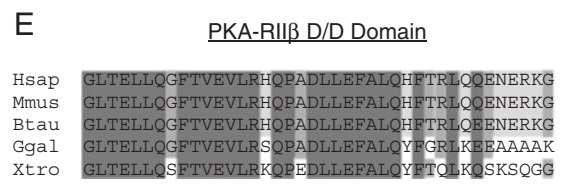

Figure 2 Amino Acid Alignments of AKAP7 RI/RII binding domain and PKA-R D/D domains. Protein regions from the AKAP7 RI/Rll binding domain (A), PKA-Rlla dimerization/Docking domain (D/D; B), PKA-Rla D/D (C) PKA-RIß D/D, (D), and PKA-RII (E) were aligned using Clustal X. (A) Residues in the AKAP7 RI/RIl binding domain that have been previously identified as components of an amphipathic a-helix necessary for PKA-R subunit interaction are indicated by $\left(^{*}\right)$ and residues that are conserved are shaded. In the AKAP7 RI/RII binding domain, a-helix forming residues are conserved in vertebrates from lamprey to humans. Residues in the PKA-Rlla (B), PKA-Rla (C), PKA-RI $\beta$ (D), and PKA-RII $\beta$ (E) D/D domains forming the docking surface for the AKAP helix which are highly conserved are indicated by shading. Residues in PKA-Rlla that are essential for AKAP binding $[48,49]$ are indicated by $\left(^{*}\right)$. The spacer region between helices in PKA-Rlla is indicated by a dotted line.

located between 7 - and $10 \mathrm{~Kb}$ upstream of the putative AKAP7y 5' exon from cow to human (Figure 4). The appearance (expression) of the AKAP7 $\delta$ splice variant coincides with the loss of expression for AKAP7y in both rat and marmoset (Calithrix jacchus) EST databases, as well as the integration of a smaller AKAP7y 5' exon in the mouse transcript which results in a truncated AKAP7 $\gamma$ amino terminus unique to mouse (Figure 4).

The sequencing of human and mouse AKAP7 $\delta$ and alignment with rat AKAP7 $\delta$ validates the presence of the multiple nucleotide mutations resulting in the truncation of AKAP7 $\gamma$ in mice and loss of AKAP7 $\gamma$ in rats (Additional file 2: Figure S2). Mouse AKAP7 $\delta$ has a 79\% nucleotide sequence identity compared to human AKAP7 8 (74\% amino acid identity), and rat AKAP7 $\delta$ has $78 \%$ nucleotide sequence identity to human AKAP7 $\delta$. Both rat and mouse AKAP7 $\delta$ splice variants contain an "RGD" amino acid sequence that is absent in human AKAP7 (Additional file 2: Figure S2).

\section{Replacement of AKAP7 $\gamma$ with AKAP7 $\delta$ indicates similar function}

Multiple sequence nucleotide alignments identified mutations in the AKAP7 genes of rat, mouse, and marmoset which result in the loss of (in rat and marmoset) or truncation of (in mouse) the AKAP7 $\gamma$ splice variant and incorporation of the AKAP7 $\delta$ splice variant
(Figure 4). In the rat AKAP7 gene, the putative AKAP7 $\gamma$ initiation codon contains two nucleotide mutations where "ATG" in the Kozak sequence is mutated to "GGG", the result of a transition at position 1 of the codon $(A \rightarrow G)$, and a transversion at position 2 of the codon $(T \rightarrow G)$ yielding the missense mutation Met $\rightarrow$ Gly. In the mouse AKAP7 gene, a cognate mutation was also identified in the putative AKAP7 $\gamma$ initiation codon as a single transition at nucleotide position $1(A \rightarrow G)$ replaces the AKAP7 $\gamma$ start codon with "GTG", resulting in the missense mutation Met $\rightarrow$ Val. Also identified in the mouse AKAP7 gene, a second mutation downstream of the AKAP7 $\gamma$ initiation codon results in the formation of an internal start site where an "ATG" is formed as the result of a nucleotide transversion $(\mathrm{C} \rightarrow \mathrm{A})$, yielding the amino acid Leu $\rightarrow$ Met mutation which yields a truncated AKAP7 $\gamma$ splice variant in mouse (Figure 4). The AKAP7y mutations in the rodent lineage seem to be specific to rats and mice, as another rodent (Oryctolagus cuniculus; rabbit) lacks these mutations, and also altogether lacks the AKAP7 5 5' exon (Figures 3 and 4). The loss of AKAP7y is also evident in marmoset, as "ATG" is replaced by "ATA", the result of a transition at position 3 of the initiation codon $(\mathrm{G} \rightarrow \mathrm{A})$ replacing Met with Ile.

Two-dimensional western analysis of recombinant rat and mouse AKAP7 long-form splice variants confirms the loss of AKAP7 $\gamma$ in rats, and shows that both 


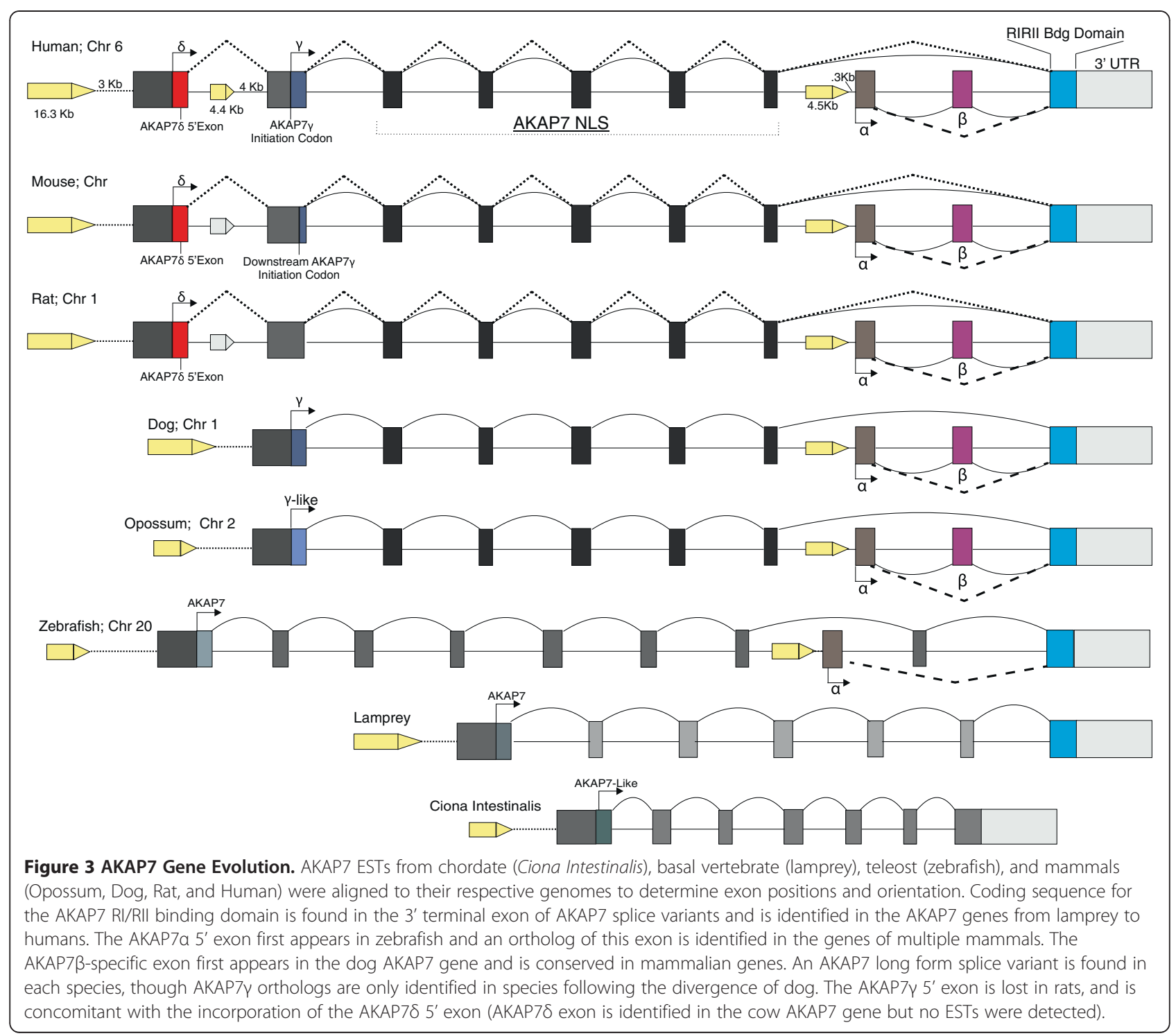

AKAP7 $\delta$ and AKAP7 $\gamma$ are produced from AKAP7 $\delta$ cDNA from mice (Figure 5). It is also apparent that mouse AKAP7 $\gamma$ is the major product from AKAP7 $\delta$ cDNA (Figure 5A), which is likely the result of an AKAP7 $\gamma$ internal translational start site that is present in mice, but absent in rats (Figure 4). AKAP7 immunoprecipitations (IPs) identified AKAP7 $\delta$ and a variant of AKAP7 $\delta$ (see below) in rat heart, while AKAP7 $\delta$, AKAP7 $\gamma$, and unknown variants were seen from mouse heart IPs (Figure 5). Comparison of rat and mouse AKAP7 cardiac localization by immunofluorescence (Figure 6) shows identical localization compared between rat (AKAP7 $\delta$ only) and mouse (predominantly AKAP7 $\gamma$ but also AKAP7 $)$ ). The pattern of AKAP7 cardiac immunstaining in rat and mouse is consistent with localization to the sarcoplasmic reticulum, as previously reported for AKAP7 $\delta$ in rat [38].
Insertion-deletions in human and Rat AKAP7 $\delta$ yield novel splice variants

Sequencing of human AKAP7 $\delta$ identified splice variants containing nucleotide sequence insertions and deletions (indels). Human AKAP7 $\delta$ variant-1, contains a seventy five nucleotide insertion between the first and second exons of AKAP7 $\delta$ (located 5' to the AKAP7y start codon; Figure 7), and an exon deletion (AKAP7 $\delta$ exon 6; Figure 7). AKAP7 $\delta$ variant- 2 contains two insertions; a sixty nine-nucleotide insertion between AKAP7 $\delta$ exons 1 and 2, and a sixty nine nucleotide insertion between AKAP7 $\delta$ exons 7 and 8 (Figure 7). The upstream insertion sequence in AKAP7 $\delta$ variant- 1 has $100 \%$ sequence identity to a region in the human AKAP7 gene (located on chromosome 6) between the AKAP7 $\delta 5$ ' exon and AKAP7 $\delta$ exon 2. The upstream insertion sequence in variant-2 has $96 \%$ sequence identity to the human 


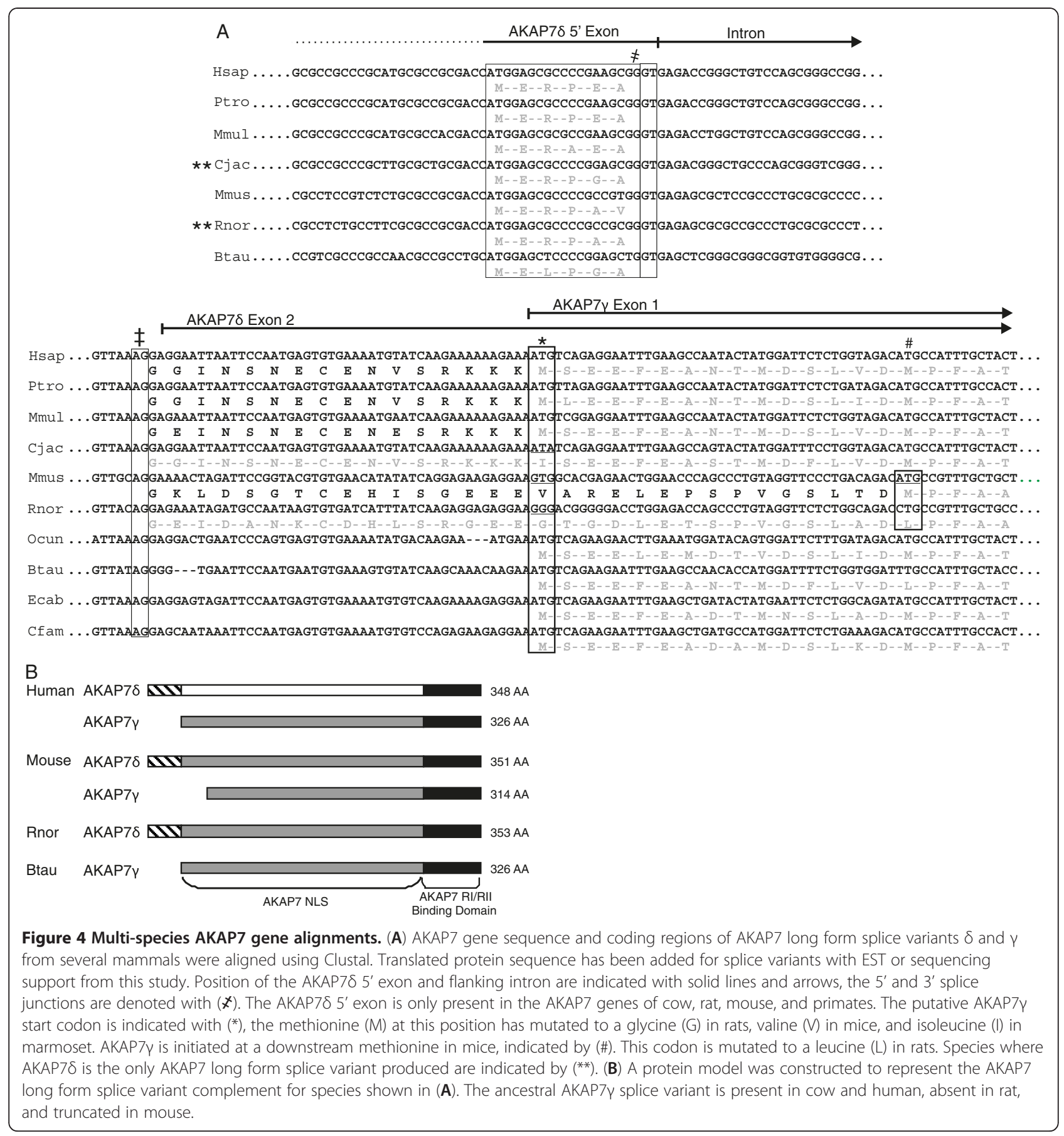

AKAP7 gene. The downstream insertion sequence in AKAP7 $\delta$ variant- 2 has $100 \%$ sequence identity to the AKAP7 $\beta$-specific sequence. PCR amplification of the human AKAP7 $\delta$ indel regions show the 5' insertion is predominant relative to the native form, while the deletion-variant is less prominent than the native transcript form (Figure 7C). The AKAP7 $\delta$ splice variant containing the AKAP7 $\beta$ exon is a minor product but is identified in most tissues (Figure 7C).
Open reading frame (ORF) analysis of Human AKAP7 $\delta$ transcript variants- 1 and 2 (Figure 8 ) show that the AKAP7 $\delta$ ' insertions shifts the reading frame downstream to begin initiation at the AKAP7 $\gamma$ coding region (Figure 8A), while the downstream exon deletion seen in AKAP7 $\delta$ variant-1 results in a truncated protein that contains the AKAP7 nuclear localization signal (NLS), but lacks the AKAP7 RI/RII binding domain (Figure 8B). The open reading frame for AKAP7 $\delta$ variant-2 begins at 


\section{A}

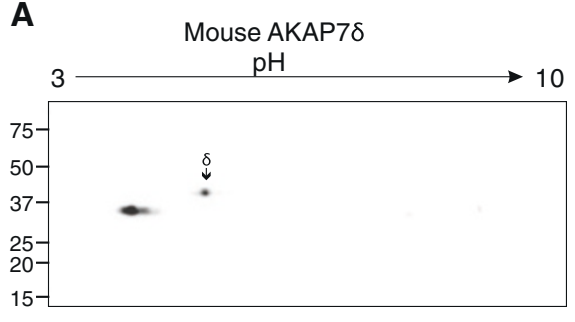

C

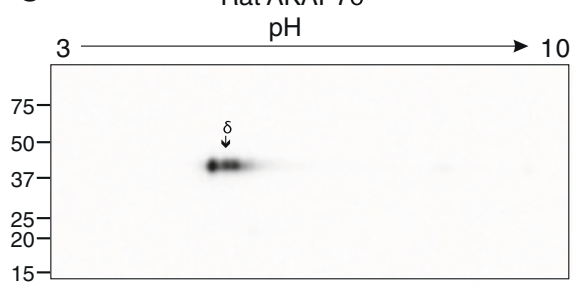

\section{E}
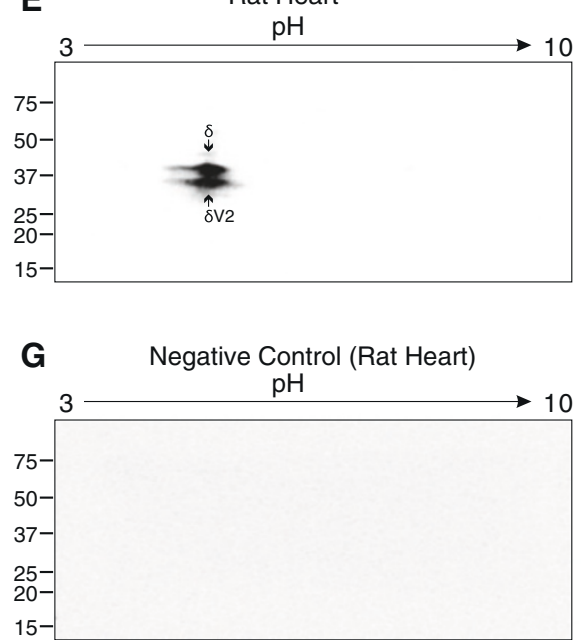

B

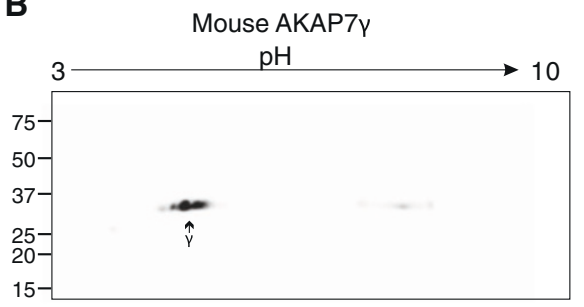

D Negative Control (HEK cells)
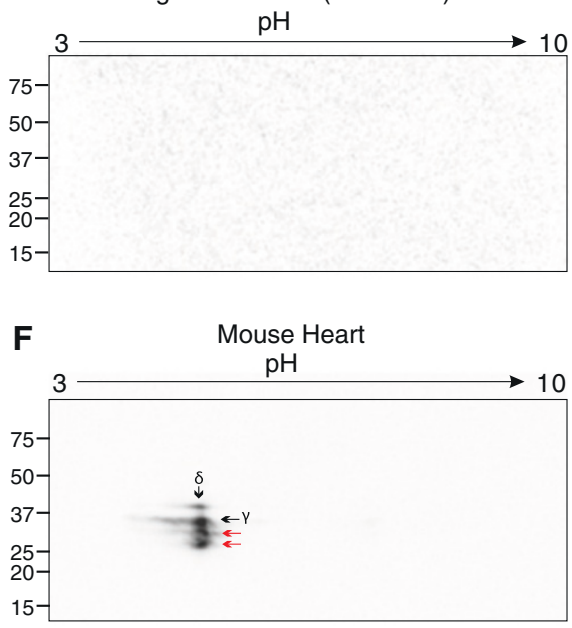

H Negative Control (Mouse Heart)

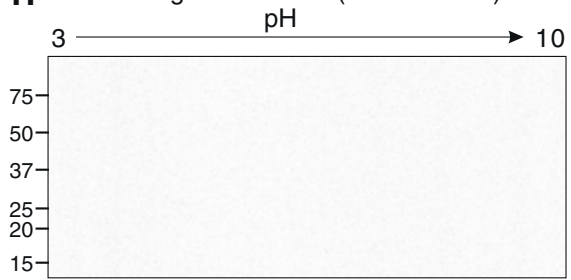

Figure 5 2D Western Analysis of AKAP7 $\delta$ and AKAP7 $\gamma$. Recombinant mouse AKAP7 $\delta$, AKAP7Y, and rat AKAP7 $\delta$ were analyzed by twodimensional western blotting. V5-tagged Mouse AKAP7 $\delta$ (A), AKAP7Y (B) or rat AKAP7 $\delta$ (C) were detected from transfected HEK293 cell lysates using a V5-HRP antibody. (D) Mock-transfected HEK293 cell lysates were probed with V5-HRP as a negative control. (E) Rat heart lysate used in AKAP7 immunoprecipitations (using AKAP7 antibody) was probed with protein A-HRP. Two products are identified, rat AKAP7 $\delta$, and a variant of rat AKAP7 $\delta$ identified in this study (rat AKAP7 $\delta$ variant 1, see Supplemental Information Figure 3). (F) Mouse heart lysate subjected to AKAP7 immunoprecipitation followed by detection with protein A-HRP. Mouse AKAP7 $\delta$ and AKAP7Y are identified in addition to two unknown splice variants (indicated by red arrows). For negative control immunoprecipitations rat heart lysate $(\mathbf{G})$ or mouse heart lysate $(\mathbf{H})$ was immunoprecipitated with rabbit lgG (pre-immune serum) followed by detection by protein A-HRP. The pH range and molecular weight are indicated at the top and left (respectively) of each blot.

the AKAP7y initiation codon and produces a protein containing the AKAP7 NLS, AKAP7 RI/RII binding domain and the AKAP7 $\beta$ protein region (Figure $8 C$ ).

Two exon-deletion transcript variants were also identified in rat AKAP7 $\delta$ (Additional file 3: Figure S3). AKAP7 $\delta$ variant-1 contains deletions at exons 3-6, while variant- 2 contains a deletion of exon 6 . Of these variants, only rat AKAP7 $\delta$ variant- 2 contains the AKAP7 RI/RII binding domain. No indels were detected from sequencing mouse AKAP7 $\delta$, however a short (3-exon) AKAP7 $\gamma$ splice variant was detected in mouse ESTs
(Acc\# BC099487.1), and is marginally expressed in mouse spleen (Additional file 4: Figure S4).

\section{Conservation in AKAP7 short splice variants}

AKAP7 short splice variants (AKAP7 $\alpha$ and $\beta$ ) have pronounced sequence conservation across the vertebrate phyla. Nucleotide sequence alignments of the AKAP7 $\alpha /$ $\beta$ 5' exon show that this exon is highly conserved from zebrafish to humans (Additional file 5: Figure S5), as the nucleotide sequence homology for this exon ranges from 66 to $98 \%$ identity. Amino acid alignment of the 


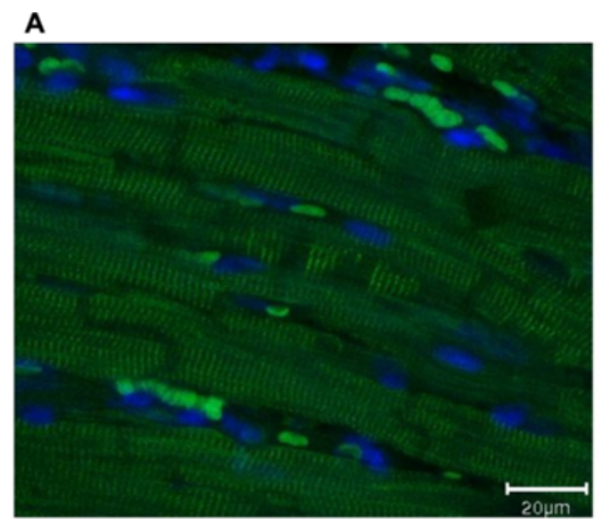

C

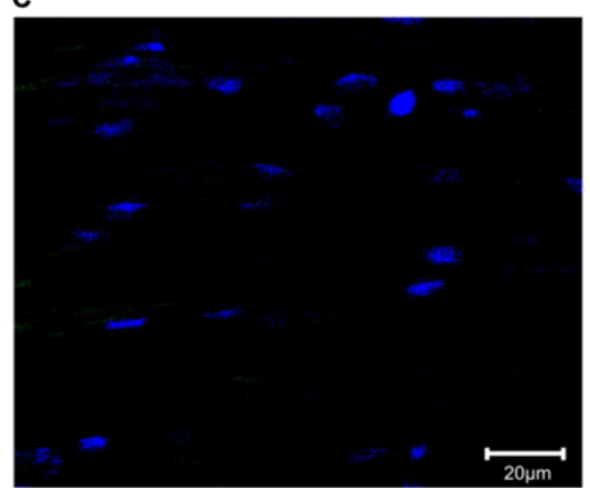

B

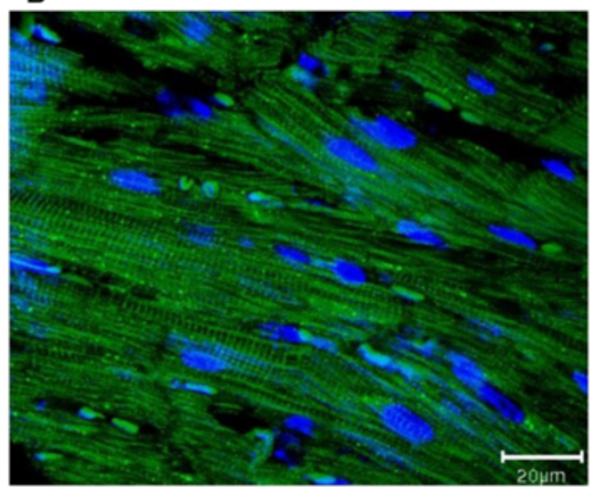

D

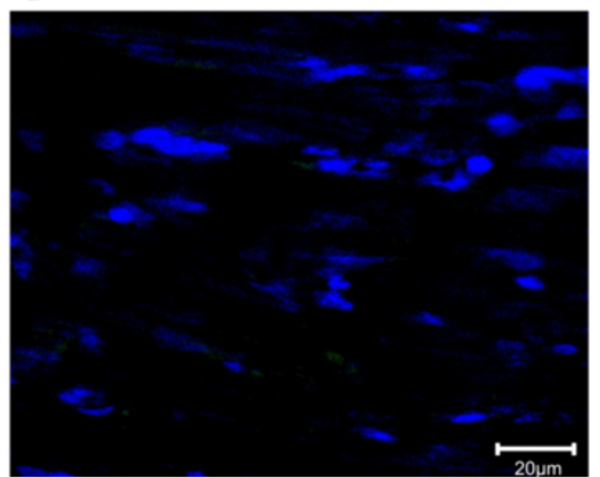

Figure 6 AKAP7 Localization in rat and mouse heart. Merged immunofluorescence images of (A) adult rat and (B) mouse hearts were taken using a confocal microscope. Immunostaining was performed using a rabbit anti-AKAP7 antibody, the $2^{\circ}$ Ab is Alexa fluor 488 anti-rabbit. Nuclei are stained with Dapi (blue). AKAP7 immunostaining is prominent in the sarcoplasmic reticulum in both rat (expressing AKAP7 $\delta$ only) and mouse (predominantly AKAP7y but also AKAP78). In the negative controls for rat (C) and mouse (D) normal rabbit serum (AKAP7 pre-immune serum) was used in place of the AKAP7 primary antibody. Images were taken at 60X magnification.

AKAP7 $\alpha / \beta$ amino terminus (Additional file 3: Figure S3) shows this protein region has a sequence homology of 70 to $100 \%$ from zebrafish to humans.

AKAP7 $\beta$ is distinguished from AKAP7 $\alpha$ by the inclusion of a 69 -nucleotide exon between the AKAP7 $\alpha / \beta 5$ ' exon and the AKAP7 3' terminal exon. This AKAP7 $\beta$ specific exon is found in the genomes of several mammals (Figure 3, Additional file 1: Figure S1), and is highly conserved (Additional file 5: Figure S5). Amino acid alignment of the AKAP7 $\beta$-specific protein region shows sequence homology of 65 to $95 \%$ from pigs to human (Additional file 5: Figure S5).

\section{Tissue Expression of AKAP7 splice variants in rodents and humans}

AKAP7 $\delta$ is ubiquitously expressed in human, mouse and rat tissues (Figure 9). AKAP7 $\alpha$ expression is expressed in several rodent and human tissues, with conserved expression seen between rat, mouse and human heart, kidney, brain, liver and lung (Figure 9). AKAP7 $\beta$ mRNA expression is also detected in multiple tissues in rodent and human, though conserved expression between rodents and humans is only seen in the kidney, with the predominant AKAP7 $\beta$ expression site is the pancreas in humans (Figure 9).

\section{Discussion}

In this study we have provided the first evolutionary analysis of an AKAP. The significance of PKA signaling has been shown throughout eukaryotic organisms. Here, we show the AKAP7 RI/RII binding domain appears in basal vertebrates, corresponding to a PKA-R subunit gene duplication event. In the molecular evolution of the AKAP7 gene, AKAP7 short splice variants appeared early in the vertebrate lineage and have remained highly conserved. In the AKAP7 long form splice variants, multiple nucleotide mutations and insertions-deletions have resulted in the concurrent loss of AKAP7 $\gamma$ and formation of AKAP7 $\delta$ in rats. Specifically in rats, the replacement of AKAP7 $\gamma$ with AKAP7 $\delta$, as shown in this study, has likely lead to an over-statement of the significance of AKAP7 $\delta$ in species other than rat $[29,38,44,50,51]$. Evolutionary pressures in the form of nucleotide insertions and mutations in the Human AKAP7 $\delta$ coding sequence 


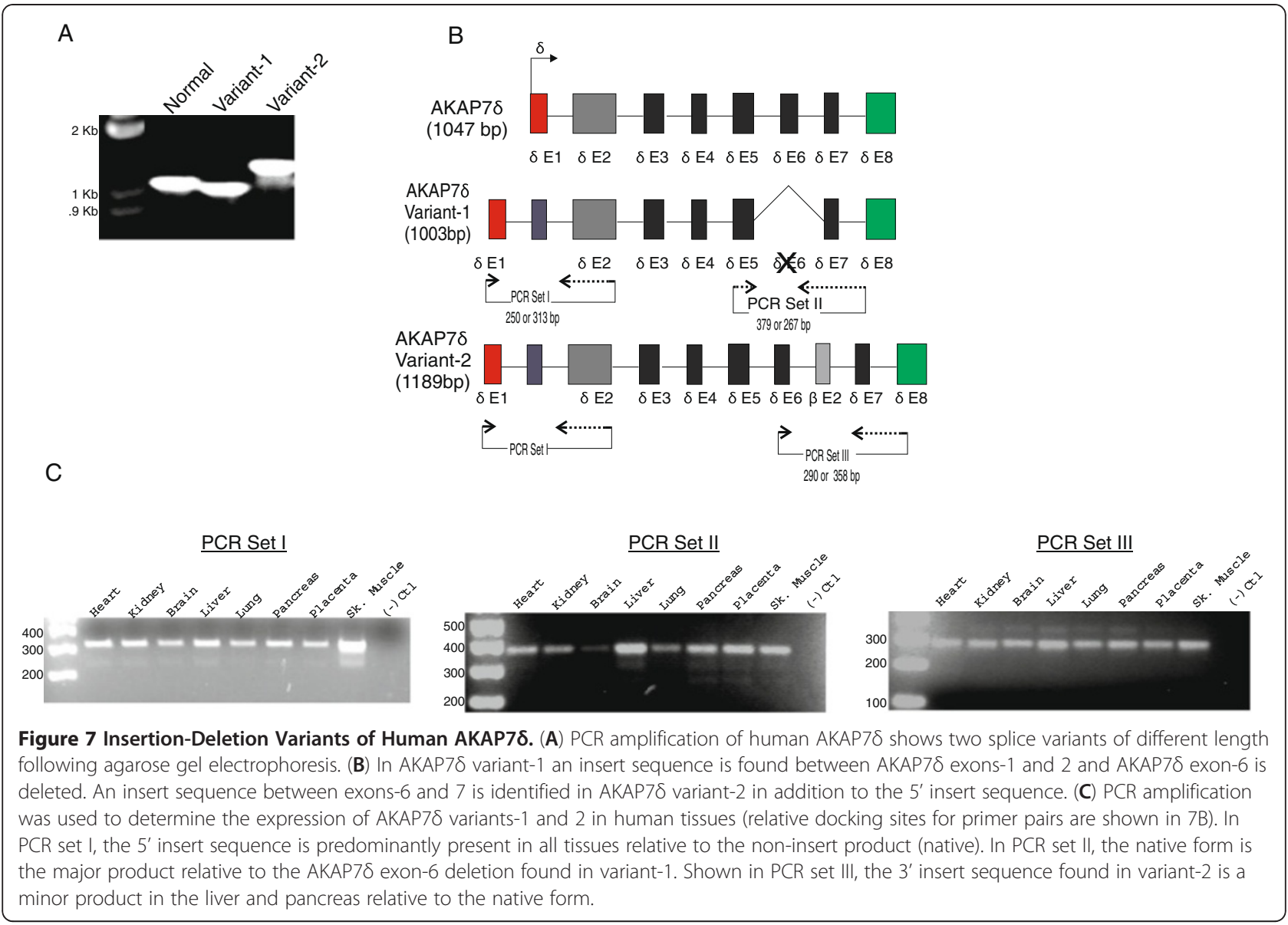

have likely driven preference for AKAP7 $\gamma$ production in humans. Hence, it is likely that AKAP7 $\delta$ is of minimal significance in human AKAP7-mediated PKA signaling.

\section{Integration of the AKAP7-RI/RII binding domain and PKA-} $R$ Dimerization/Docking domain in vertebrate evolution While AKAP7-like proteins were identified in invertebrate and chordate species, the appearance of the RI/RII binding domain (and formation of AKAP7 proteins) occurs in basal vertebrates (lamprey). The formation of AKAP7 proteins from AKAP7-like proteins corresponds with PKA-R gene duplication events where the PKA-RI gene duplicates to form PKA-RI $\alpha$ and PKA-RI $\beta$, and PKA-RII forms PKA-RII $\alpha$ and PKA-RII $\beta$. Evolution of the PKA-R subunits has been previously described [52]. Here we provide greater resolution of the timing of PKA-R gene duplications, and show that the amino acid sequences of PKA-R D/D domains are highly conserved, even prior to the gene duplication events. The relative fixation of PKA-R D/D sequences prior to and following the appearance of the AKAP7 RI/RII binding domain suggests that AKAP7 evolved subsequent to the increased presence of PKA (the result of PKA-R gene duplications). This is supported by the relatively rapid development of the AKAP7 RI/RII binding domain which is absent in chordates (ciona), but present in vertebrates (lamprey).

\section{Origin of AKAP7 $\delta$, the need for an AKAP7 long-form splice variant}

As seen in our AKAP7 gene and splice variant phylogenetic analyses, AKAP7 $\gamma$ is the ancestral AKAP7 long form splice variant in mammals. Several nucleotide mutations identified in the murine lineage (as well as a basal primate) resulted in the loss of AKAP7 $\gamma$ through mis-sense mutations in the translational start codon. The most dramatic of these mutations occurs in rats, where the AKAP7 $\gamma$ initiation codon contains a successive transition and rare transversion that results in glycine replacing methionine. The results of these mutations have been confirmed in NCBI database searches, as rat AKAP7y has been removed from the database (acc. \#XM_001053676.1 has been removed), mouse AKAP7 $\gamma$ is truncated (acc. \#NM_018747.4) and marmoset EST search results show AKAP7 $\gamma$ is not present whereas AKAP7 $\delta$ is expressed (accession number XM_002746958.1). The combination of the AKAP7 $\gamma$ initiation codon loss combined with the 


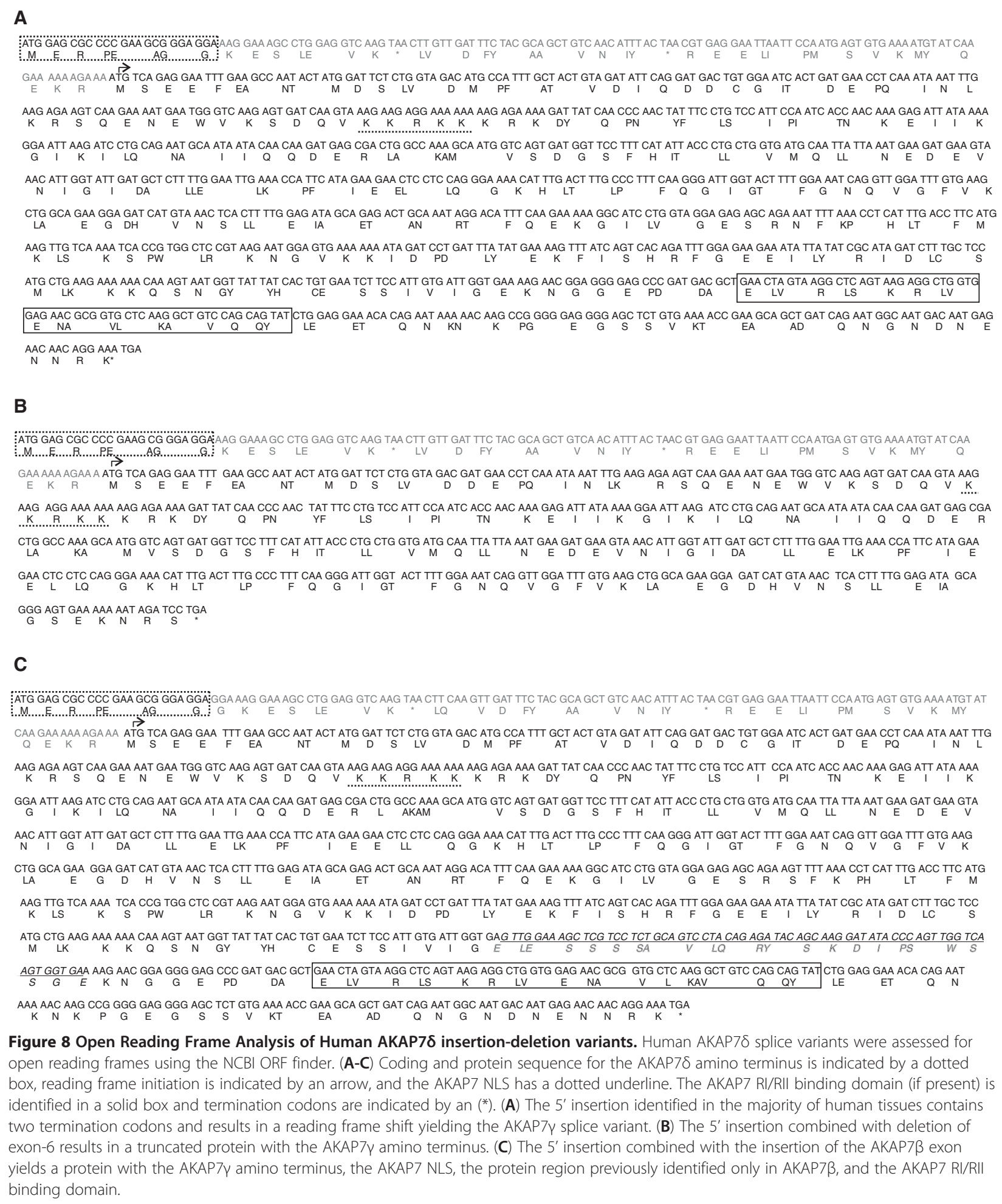

Figure 8 Open Reading Frame Analysis of Human AKAP7 $\delta$ insertion-deletion variants. Human AKAP7 $\delta$ splice variants were assessed for open reading frames using the NCBI ORF finder. $(\mathbf{A}-\mathbf{C})$ Coding and protein sequence for the AKAP7 $\delta$ amino terminus is indicated by a dotted box, reading frame initiation is indicated by an arrow, and the AKAP7 NLS has a dotted underline. The AKAP7 RI/RII binding domain (if present) is identified in a solid box and termination codons are indicated by an $\left(^{*}\right)$. (A) The $5^{\prime}$ insertion identified in the majority of human tissues contains two termination codons and results in a reading frame shift yielding the AKAP7Y splice variant. (B) The $5^{\prime}$ insertion combined with deletion of exon- 6 results in a truncated protein with the AKAP7Y amino terminus. (C) The $5^{\prime}$ insertion combined with the insertion of the AKAP7 $\beta$ exon yields a protein with the AKAP7Y amino terminus, the AKAP7 NLS, the protein region previously identified only in AKAP7 3 , and the AKAP7 RI/RII binding domain.

concurrent absence of a downstream initiation codon utilized in mice (and present in the AKAP7 genes of several other species), likely yielded the evolutionary pressure for the incorporation of the AKAP7 $\delta 5^{\prime}$ exon in rats.
In vitro analyses of mouse AKAP7 $\gamma, \mathrm{AKAP7} \delta$ and rat AKAP7 $\delta$ show that both $\gamma$ and $\delta$ splice variants are produced from the mouse AKAP7 $\delta$ transcript, suggesting that $\mathrm{AKAP} \gamma$ is an internal translational start site product of AKAP7 $\delta$ production. This is consistent with our 


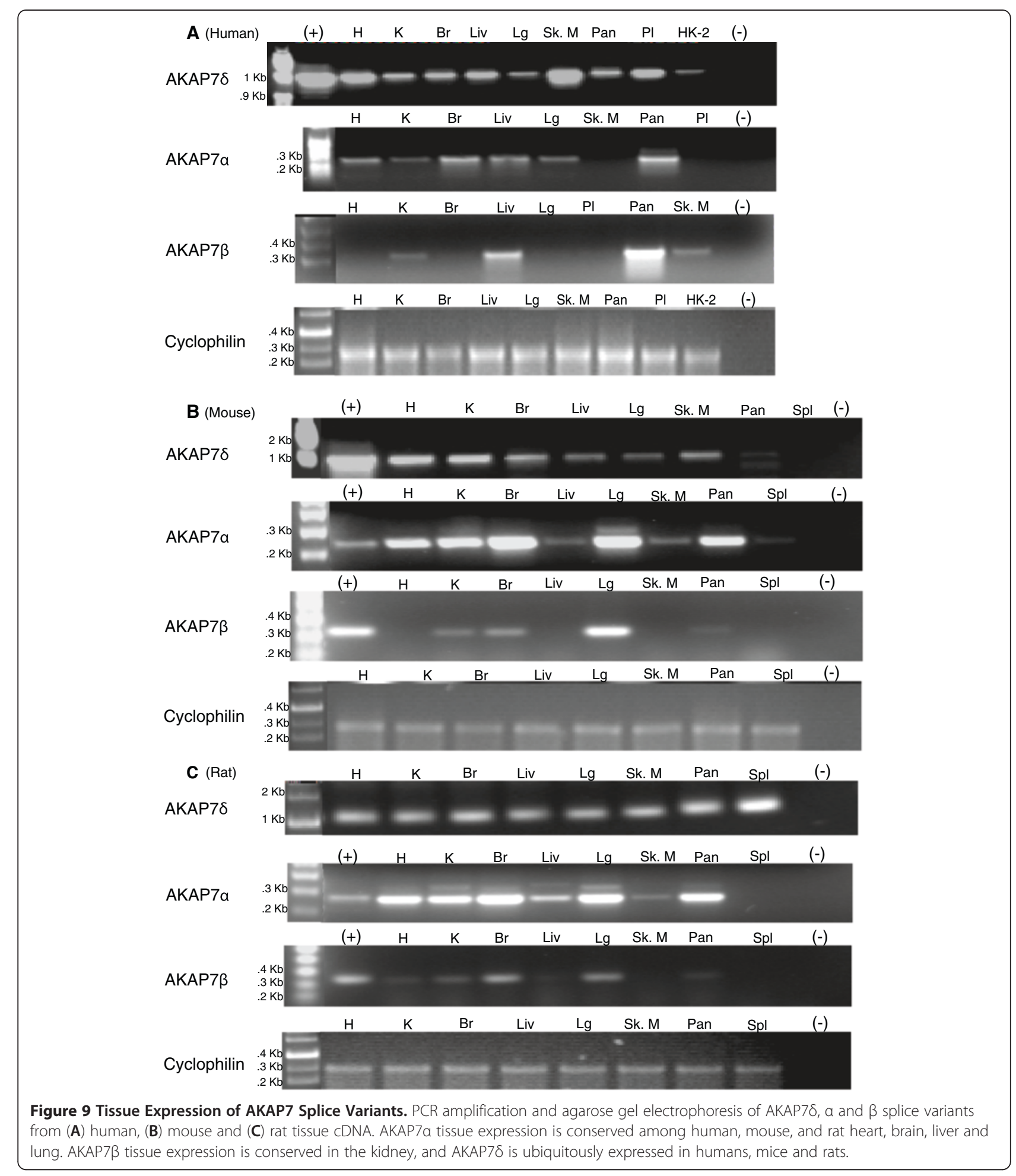

AKAP7 gene analysis and provides a different model for AKAP7 long form splice variants than have been previously reported [29,53]. Our results also suggest that AKAP7 $\gamma$ is the major product of AKAP7 $\delta$ transcription and translation. In rats, only a single protein product was identified from recombinant AKAP7 $\delta$ expression which is consistent with gene and EST alignments showing the loss of AKAP7 $\gamma$ in rats. Findings from previous studies that have addressed AKAP7 $\gamma$ synthesis and function in rats $[53,54]$ should be solely attributed to AKAP7 $\delta$ as the AKAP7 $\gamma$ splice variant is absent in rats. The significant role of AKAP7 $\delta$ in the renal aquaporin 
shuttle [29], and regulation of cardiac $\mathrm{Ca}^{2+}$ cycling [38] has led to a concerted effort to develop AKAP7 $\delta$ peptidomimetics for use in treating human heart failure $[44,50]$. Results from this study show that AKAP7 $\delta$ is the sole AKAP7 long form splice variant found in rats, and the significance of this splice variant is very likely over-stated. Combined with the identification of nucleotide insertions in the 5' end of human AKAP7 $\delta$ that would exclusively promote the production of AKAP7 $\gamma$ in all tissues, the evidence presented here indicates that the functional significance of AKAP7 $\delta$ is minimal in species other than rats. In mice, where AKAP7 $\gamma$ appears to be the dominant product of AKAP7 $\delta$ transcription and translation, cardiac AKAP7 immunlocalization is consistent with the SR indicating AKAP7y largely replaces AKAP7 $\delta$ at the SR in species other than rat and supports the possibility that AKAP7 $\delta$ and AKAP7y are functionally redundant in mice. Further studies are required to determine the functional consequences and distinction between AKAP7 $\delta$ and AKAP7 $\gamma$ in mice and human skeletal muscle.

Evolutionary pressure for replacing AKAP7 $\delta$ with AKAP7Y AKAP7 $\delta$ has been identified as a vital component in cardiac $\mathrm{Ca}^{2+}$ handling at the sarcoplasmic reticulum in rats [38] and has been proposed as a pharmacological target in treating heart failure $[44,50]$. Our evolutionary analysis of the AKAP7 gene has shown that the origin of AKAP7 $\delta$ was brought about through a series of nucleotide mutations in the AKAP7 $\gamma$ coding sequence in rats, hence likely leading to exaggeration of the functional significance of AKAP7 $\delta$. This is supported by in vitro analysis of mouse AKAP7 $\delta$, where AKAP7y (through a likely internal translation initiation site) is the major product. AKAP7 immunoprecipitations from rat and mouse heart tissue also show AKAP7 $\delta$ and an AKAP7 $\delta$ variant in rat while in mouse heart AKAP7 $\delta$ is a minor product and AKAP7 $\gamma$ is prominent. Furthermore, sequencing of human AKAP7 $\delta$ (this study) showed the dominant presence (in multiple tissues) of a nucleotide sequence insertion immediately downstream of the AKAP7 $\delta$ 5' codon, resulting in a reading-frame shift that promotes the production of AKAP7 $\gamma$. Therefore AKAP $\gamma$ is the predominant, functional AKAP7 long form splice variant in humans and pharmacological efforts in designing AKAP7 $\delta$ disruptor peptides should instead be directed at AKAPY.

\section{AKAP7 short form splice variant evolution}

Found only in the vertebrate lineage, AKAP7 $\alpha$ is the ancestral AKAP7 splice variant and has conserved tissue expression in rodents and human heart, kidney, brain, liver and lung. AKAP7 $\alpha$ has been extensively studied in the heart and brain, where this protein regulates PKA localization to (and phosphorylation of) ion channels such as the L-type $\mathrm{Ca}^{2+}$ channel $[1,40,55,56]$ and epithelial $\mathrm{Na}^{+}$channels $[2,36,37,41]$. Deletion of a specific region in the cardiac voltage-sensitive $\mathrm{Ca}^{2+}$ channel abolishes AKAP7 binding leading to cardiac hypertrophy and premature death in mice, and demonstrates the importance of AKAP7 $\alpha$ mediated PKA signaling in adrenergic stimulation [57]. As seen in the expression results in this study, it is also likely that AKAP7 $\alpha$ has a conserved role in ion channel regulation in kidney, liver and lung. AKAP7 $\alpha$ amino-terminal amino acids that have been previously identified [1] in membrane targeting through myristolation (glycine at position 2; Figure 9) or palmitoylation (cysteins at positions 5 and 6; Figure 9) are absolutely conserved from zebrafish to humans. Furthermore, hydrophobic residues (leucine-4, phenylalanines-7 and 9, arginine-11) in the AKAP7 $\alpha$ amino terminus which are likely to be critical for membrane localization are also conserved between zebrafish and humans. Given the high degree of sequence conservation in the AKAP7 $\alpha$ amino terminus, it is likely this protein functions in membrane ion channel regulation throughout vertebrate species following the divergence of lamprey.

Development of the AKAP7 $\beta$ exon occurred in mammals (opossum), and while AKAP7 $\beta$ and $\alpha$ splice variants share the same amino terminus, the AKAP7 $\beta$-specific exon confers a cellular localization distinct from the AKAP7 $\alpha$ splice variant [46]. The functional significance of the AKAP7 $\beta$ splice variant in mammals remains unknown, though the sequence conservation in this protein is certainly suggestive of its importance. AKAP7 $\beta$ is primarily conserved in the kidney of rodents and human, and it seems likely that this splice variant has a conserved function in renal ion channel regulation. Importantly, in this study we have identified a novel AKAP7 longform splice variant containing the $\beta$-exon. Further studies are required to address the unique membrane-localizing function of this AKAP7 protein region.

\section{Conclusions}

Our results show that the formation of AKAP7 proteins occurred in basal vertebrate species, likely in response to the increased presence and distribution of intracellular PKA following PKA-R gene duplication events. Short form splice variants from AKAP7 genes have remained highly conserved across species, while long forms have undergone rapid evolutionary change. Independent mutations in the AKAP7 genes of rats, mice and marmoset have resulted in loss or truncation of AKAP7y and a likely compensatory incorporation of AKAP7 $\delta$. These results suggest that studies of AKAP7 $\delta$ are not necessarily comparable among rats, mice and humans. 
The specific targeting of human AKAP7 $\delta$ in pharmacotherapeutics should be undertaken with caution, as this splice variant is largely absent in humans.

\section{Methods}

\section{Sequence data acquisition}

Human and rodent AKAP7 splice variant sequences were obtained from NCBI and ENSEMBL (release 61) databases by searching for the gene "AKAP7" and AKAP7 transcripts. Annotated AKAP7 proteins were analyzed for conserved AKAP7 protein families (Pfams) using both the NCBI Conserved Domain Database (CDD) and Sanger Institute Protein Family Database (http://pfam.sanger.ac.uk/) [58]. Conserved AKAP7 Pfams were identified as the AKAP7 Nuclear Localization Signal (AKAP7 NLS; Pfam 10469) and the AKAP7 RI/RII binding domain (pfam 10470). Human, rat and mouse AKAP7 Nucleotide and protein sequences obtained from NCBI (http://www.ncbi.nlm. nih.gov) and ENSEMBL were used in BLASTP, TBLASTN and EST search algorithms to identify AKAP7 transcripts, proteins and genes in multiple species (listed below). Search results were verified to contain both AKAP7 Pfams (AKAP7 NLS and AKAP7 RI/ RII). Sequences obtained from these searches that contained only the AKAP7 NLS Pfam were classified as "AKAP7-like proteins. Multiple species spanning the vertebrate and invertebrate lineages were used for this study, specifically: primates-Homo sapiens (H. sap), Pan trogylodytes (P.tro), Macaque mulatta (M. mul) Callithrix jacchus (C. jac), rodents-Mus musculus (M. mus), Rattus norvegicus (Rnor), Oryctolagus cuniculus (O. cun); laurasiatheria- Canis familiaris (C. fam), Equus caballus (E. cab), Bos Taurus (B. tau), Sus scrofa (S. scr), metatheria- Monodelphis domestica (M. dom), reptilesAnolis carolinensis (A. car), amphibians- Xenopus tropicalis (X. tro), teleosts- Danio rerio (D. rer), Takifugu rubripes (T. rub), Oryzias latipes (O. lat), Gasterosteus aculeatus (G. acu); vertebrates- Petromyzon marinus (Pmar); chordates- Ciona intestinalis (C. int).

\section{Development of PKA-R and AKAP7 interaction}

To determine the relationship between AKAP7 RI/RII binding domain and PKA-R gene evolution, we constructed a PKA-R phylogenetic tree from the PKA-R cAMP binding domains, and identified AKAP7 RI/RII binding domain presence/absence for each species used in the PKA-R phylogenetic tree. PKA-R translated amino acid sequences were aligned using the Clustal function of Mega v5 [59]. PKA-R subunit protein relationships were determined using the algorithm of Mr. Bayes 3.1.2. Mr. Bayes analysis was run under a fixed rate Dayhoff model. The tree is a representative of 4 independent runs, of four million generations each. The tree was rooted with Drosophila melanogaster. Pictoral representation of AKAP7 RI/RII presence/absence is shown next to the PKA-R clades that contain this motif.

\section{EST to Gene alignments}

To determine AKAP7 splice variant exon positions and gene structures, the AKAP7 genes of human, rat, dog, opossum, zebrafish, lamprey, and ciona were analyzed using GENESCAN [60] and Promoter 2.0 Prediction Server [61] FPROM (Softberry, Inc., Mt Kisco, NY) programs. AKAP7 splice variant exon positions and splice junctions were verified by using NCBI two-sequence BLAST (bl2seq) and manually aligning cDNA sequences to the genomic sequences using Mega v5 [59].

\section{Animals}

All experiments involving animal subjects were carried out with the approval of the University of IllinoisChicago (UIC) and Jesse Brown VA Medical Center (JBVAMC) Institutional Animal Care and Use Committees (IACUC). Adult male rats and mice were used for cardiac and renal tissue preparations in DNA sequencing, western blotting and immunofluorescence applications.

\section{AKAP7 Splice variant detection and sequencing}

Human multiple tissue cDNA (MTC panel I, CloneTech, CA) was used in PCR amplification and sequencing applications. Rat and mouse total RNA were prepared using Trizol reagent (Invitrogen). Five micrograms of total RNA was used in first-strand cDNA synthesis using SuperScript III Reverse Transcriptase (Invitrogen) primed with random hexamers. PCR was performed using KOD Hot Start DNA polymerase (EMD Biosciences). Primers used for the amplification of human, mouse and rat $\mathrm{AKAP} 7 \alpha, \beta$, and $\delta$ splice variants are listed in Table 1. PCR products were extracted from agarose gels and cloned into the pGEM-T vector system (Promega) and sequenced using a capillary sequencer (Applied Biosystems).

\section{Transfections}

Mouse AKAP7 $\delta, A K A P 7 \gamma$ and rat AKAP7 $\delta$ transcripts were PCR amplified from heart or kidney cDNA templates and cloned into a pcDNA3.1/V5-His expression vector (Invitrogen) to produce V5-tagged proteins. For transient transfection purposes, 2x10 ${ }^{5}$ HEK293 cells (American Tissue Culture Collection) were grown on 6well plates for $24 \mathrm{~h}$ in Dulbecco's Modified Eagle Medium, 10 \% Fetal Bovine Serum (FBS) (BioWhitaker), $100 \mathrm{U} / \mathrm{mL}$ penicillin and $50 \mu \mathrm{g} / \mathrm{mL}$ streptomycin. Cell transfections were performed by using Lipofectamine 2000 (Invitrogen Life Technologies) according to the manufacturer's instructions. Cells were transfected with 
Table 1 Primers used in PCR amplifications and Sequencing

\begin{tabular}{|c|c|}
\hline Name & Sequence $5^{\prime}$ to $3^{\prime}$ \\
\hline Human AKAP7S For & ATGGAGCGCCCCGAAGCGGGAGGAATTAAT \\
\hline Human pan AKAP7 Rev & TCATTTCCTGTTGTTCTCATTGTCATTGCCATTCTGATCA \\
\hline Human AKAP7a For & ATGGGCCAGCTTTGCTGCTTTCCTTTCTCAAG \\
\hline Human AKAP7ß For & GAGTTGGAAAGCTCGTCCTCTGCAGTCCTAC \\
\hline Human AKAP78-indel set 1 For & ATGGAGCGCCCCGAAGCGG \\
\hline Human AKAP78-indel set 1 Rev & TTTCCTCTTCTTTACTTGATCACTCTTGACCCATTCATT \\
\hline Human AKAP7反-indel set 2 For & TTCATAGAAGAACTCCTCCAGGGAAAACATTTGACTITG \\
\hline Human AKAP78-indel set 2 Rev & GAAGATTCACAGTGATAATAACCATTACTTTGTTT \\
\hline Human AKAP7反-indel set 3 For & CCTGGTAGGAGAGAGCAGAAGTITAAAACCTCATTTGAC \\
\hline Human AKAP7反-indel set 3 Rev & TCCACCAGCCTCTTACTGAGCCTTACTAGTTC \\
\hline Mouse AKAP78 For & CACCATGGAGCGCCCCGCCGT \\
\hline Mouse pan AKAP7 Rev & CTTCCGGTTGTTATCACTGCCATCACCATTCCGAT \\
\hline Mouse AKAP7Y For & CACCATGCCGTTTGCTGCTGTAGATATTCAAGATGA \\
\hline Mouse AKAP7a For & ATGGGCCAGCTTTGCTGCTTCCCTTTCG \\
\hline Mouse AKAP7 $\beta$ For & GAGTTGGAAAATCCATCTTCTGCAGACCTACAGAG \\
\hline Mouse AKAP7Y-short Rev & TTAGTGCCCAGGGTGGAGCTTTGGAGCAGT \\
\hline Rat AKAP7 $\delta$ For & CACCATGGAGCGCCCCGCCGCGGGA \\
\hline Rat pan AKAP7 Rev & CTTCCGGTTGTTATCACTGCCATCGCCATTCC \\
\hline Rat AKAP7a For & ATGGGCCAGCTTTGCTGCTTCCCC \\
\hline Rat AKAP7 $\beta$ For & CAGTTGGAAAGTCCATCTTCTGCAATCCTACAGAGAT \\
\hline
\end{tabular}

mouse AKAP7 $\delta$, mouse AKAP7 $\gamma$, or rat AKAP7 $\delta$. Transfected and mock-transfected cells were harvested 48 hours after transfection and washed with PBS followed by sonication in deionized urea-thiourea-chaps buffer (UTC; $8 \mathrm{M}$ urea, $2 \mathrm{M}$ Thiourea, $2 \%$ chaps) with protease and phosphatases inhibitors (Pierce). Supernatants were then used in 2D western applications.

\section{Immunoprecipitations}

Immediately following removal, rat and mouse hearts were fast-frozen in liquid nitrogen and dounce homogenized at $4^{\circ} \mathrm{C}$ in $50 \mathrm{mmol} / \mathrm{L}$ of Tris- $\mathrm{HCl}(\mathrm{pH} \mathrm{7.4)}$, $150 \mathrm{mmol} / \mathrm{L}$ of $\mathrm{NaCl}, 1 \mathrm{mmol} / \mathrm{L}$ of EDTA, $0.25 \%$ sodium deoxycholate, $1 \%$ triton X-100 and $1 \mathrm{X}$ HALT protease and phosphatases inhibitor cocktails (Pierce). The homogenate was centrifuged at 10,000X RCF for 15 minutes at $4^{\circ} \mathrm{C}$, the supernatant was collected and protein concentrations were determined using the Dc Protein Assay (BioRad). Five hundred micrograms of the heart lysates were incubated in $500 \mu \mathrm{l}$ lysis buffer containing $1 \mu \mathrm{g}$ of rabbit polyclonal AKAP7 antibody with slow rotation on a rocker overnight at $4^{\circ} \mathrm{C}$. Negative control immunoprecipitations were carried out using rabbit IgG (pre-immune serum) instead of the AKAP7 antibody. Lysate-immunoglobulin samples were then incubated with $50 \mu \mathrm{l}$ of protein $\mathrm{A} / \mathrm{G}$ agarose beads (EMD) for 2 hours under constant rotation. The samples were then centrifuged, supernatants were removed and agarose bead pellets were successively washed in PBS containing $1.00 \%, 0.5 \%$, and $0.05 \%$ Tween-20. The beads were resuspended in deionized UTC buffer with protease and phosphatases inhibitors, mixed and incubated at room temperature for 10 minutes. Following centrifugation, the supernatants were used in 2D Western applications.

\section{Two-dimensional western analysis}

Samples from transfections and heart lysate immunoprecipitations were centrifuged for 10 minutes at 10,000 RCF and the protein content of the supernatants was estimated using the RC/DC protein assay (BioRad). Fifty micrograms of total protein was isoelectrically focused (IEF) using $7 \mathrm{~cm}$ isolated $\mathrm{pH}$ gradient (IPG, pH 3-10 non-linear gradient) strips on an IEF cell (BioRad). Second-dimensional separations were performed on 4$15 \%$ gradient tris-glycine SDS-PAGE gels. Proteins were transferred to PVDF membranes for western analysis. To detect recombinant AKAP7 proteins from transfections, membranes were probed using an anti-V5 HRPconjugated antibody (Invitrogen) at a 1:5000 dilution. Proteins from AKAP7 immunoprecipitations were detected with a protein-A HRP conjugate. In negative control immunoprecipitations, rabbit IgG was used in 
place of the AKAP7 antibody. Western blot images were taken on a Kodak image station 4000R pro.

\section{Immunofluorescence microscopy}

Affinity-purified rabbit polyclonal pan-AKAP7 antisera was produced by Genscript corp. (Piscataway, NJ) for use in immunoprecipitations and immunofluorescence applications in rat and mouse hearts. The epitope used for AKAP7 antisera production was "LVRLSKRLVENAVC" which is common to rodent and human AKAP7 splice variants. Following removal, rat and mouse hearts were fixed overnight with $10 \%$ formalin in $0.1 \mathrm{M}$ phosphate buffer ( $\mathrm{pH}$ 7.2). Tissues were dehydrated in graded ethanol solutions and embedded in paraffin. Nonspecific staining was blocked by incubation with $1.5 \%$ normal goat serum in PBS for $1 \mathrm{hr}$ at room temperature in a humidified atmosphere. The sections were incubated with the anti-AKAP7 antibody or normal rabbit serum (for negative control) overnight at $4 \mathrm{C}$. After three washes of 10 min each in PBS-T, the sections were incubated with Alexa fluor 488-conjugated anti-rabbit secondary antibody (Invitrogen) for 2 hours at room temperature and washed three times. Nuclei were counterstained with Dapi.

\section{Additional files}

Additional file 1: Figure S1. AKAP7 Splice Variant Evolution. EST

classification and EST to gene matching shows the differential incorporation of AKAP7 splice variants throughout evolution. A

cladogram was constructed using the NCBI taxonomy common tree tool and matches known species phylogenetic relationships. The accession numbers from NCBI or Ensemble databases and corresponding exonintron structures in each species (determined by EST to gene matching) for the AKAP7 splice variants is shown in each column. A splice variant is indicated as "not present" if the exon(s) for that splice variant are absent from the AKAP7 gene. A splice variant is indicated as "not detected" if the necessary exons are identified in the AKAP7 gene but no EST was identified. Splice variants with a non-orthologous AKAP7Y exon-1 are listed as AKAP7Y-like.

Additional file 2: Figure S2. Nucleotide and Amino acid alignments of human, mouse and rat AKAP78. (A) Human and mouse AKAP7 $\delta$ splice variants were sequenced and aligned to the previously reported rat AKAP7 $\delta$ nucleotide sequence. Predicted mutations in the AKAP7Y initiation codon are confirmed, and indicated by an (*). There is $78-89 \%$ sequence identity between rat, mouse, and human AKAP7 $\delta$ nucleotide sequence. The least similar region is found between nucleotides 180 and 200, (B) translated amino acid sequences were aligned using ClustalX2. Rat and mouse AKAP7 $\delta$ contain an "RGD" domain between amino acids 70 and 75 , this domain is absent from the human sequence.

Additional file 3: Figure S3. Splice variants of Rat AKAP78. (A) PCR amplification of AKAP7 $\delta$ from rat heart CDNA yielded two smaller splice variants in addition to the native form. (B) Rat AKAP7 $\delta$ variant- 1 has an exon-7 deletion, whereas AKAP7 $\delta$ variant- 2 consists of exons 1, 2 and 8 , while exons 3-7 are deleted. Rat AKAP7 $\delta$ variant-1 contains the AKAP7 NLS (dotted underline) but not the AKAP7 RI/RII binding domain. Rat AKAP7 $\delta$ variant- 2 lacks the AKAP7 NLS but contains the AKAP7 RI/RII binding domain (box).

Additional file 4: Figure S4. Mouse AKAP7Y splice variant. A short splice variant consisting of AKAP7Y exons 1 and 2 , and a unique $3^{\prime}$ exon was identified in mouse ESTs. (A) This truncated splice variant contains the AKAP7 NLS (dotted box), but lacks the AKAP7 RI/Rll binding domain. A model was constructed (B) to show the relative positions of constituent exons. (C) The mouse AKAP7y short variant is not widely expressed, and is detected only in the spleen.

Additional file 5: Figure S5. Nucleotide and amino acid alignments of AKAP7 short form splice variants. (A) The amino terminus of AKAP7 $a$ and AKAP7 $\beta$ was aligned using clustalX2. Amino acid sequences are highly conserved from zebrafish to humans. Residues that have been previously identified as lipid-modified [1] are indicated by (\$) and hydrophobic residues are indicated with down-arrows ( $\downarrow$ ). (B) The AKAP7ß-specific region is identified in species from pig to humans, with several highly conserved residues which are indicated with $\left(^{*}\right)$.

\section{Abbreviations}

AKAP: A-Kinase Anchoring Protein; PKA-R: PKA regulatory subunit; PKA-R D/ D: PKA regulatory subunit dimerization/docking domain; SERCA: Sarcoplasmic-Endoplasmic Reticulum Calcium ATPase;

PLN: Phospholamban; ORF: Open Reading Frame..

\section{Competing interests}

The authors declare that they have no competing interests

\section{Authors' contributions}

KRJ conceived the study, designed the experiments, obtained data, interpreted data, and drafted the manuscript. JNJ constructed the PKA-R phylogenetic tree and edited the manuscript. GKC provided technical advice and edited the manuscript. RSD edited the manuscript and supported study. All authors have read and approved the manuscript for publication.

\section{Acknowledgements}

This study was supported in part by National Institutes of Health Institutional NRSA training grant NIH T32 HL 07692 (KRJ) and Veterans Affairs (VA) Merit Award (RSD).

\section{Author details}

'Department of Medicine, University of Illinois at Chicago, Chicago, IL, USA. 2Department of Human Genetics, University of Chicago, Chicago, IL, USA. ${ }^{3}$ Department of Pharmacology, University of Illinois at Chicago, Chicago, IL, USA. ${ }^{4}$ Jesse Brown VA Medical Center, Chicago, IL, USA. ${ }^{5}$ Department of Cardiology, University of Illinois at Chicago, 840S. Wood Street, Chicago, IL 60612, USA.

Received: 30 December 2011 Accepted: 10 July 2012

Published: 26 July 2012

\section{References}

1. Fraser ID, et al: A novel lipid-anchored A-kinase Anchoring Protein facilitates CAMP-responsive membrane events. EMBO J 1998, 17(8):2261-2272.

2. Tibbs VC, et al: AKAP15 anchors CAMP-dependent protein kinase to brain sodium channels. J Biol Chem 1998, 273(40):25783-25788.

3. Paulucci-Holthauzen $A A$, et al: Spatial distribution of protein kinase $A$ activity during cell migration is mediated by A-kinase anchoring protein AKAP Lbc. J Biol Chem 2009, 284(9):5956-5967.

4. Carnegie GK, Scott JD: A-kinase anchoring proteins and neuronal signaling mechanisms. Genes Dev 2003, 17(13):1557-1568.

5. Banky $P$, et al: Related protein-protein interaction modules present drastically different surface topographies despite a conserved helical platform. J Mol Biol 2003, 330(5):1117-1129.

6. Newlon MG, et al: The A-kinase anchoring domain of type llalpha CAMPdependent protein kinase is highly helical. J Biol Chem 1997, 272(38):23637-23644.

7. Newlon MG, et al: A novel mechanism of PKA anchoring revealed by solution structures of anchoring complexes. EMBO J 2001, 20(7):1651-1662.

8. Fink MA, et al: AKAP-mediated targeting of protein kinase a regulates contractility in cardiac myocytes. Circ Res 2001, 88(3):291-297.

9. Potet $F$, et al: AKAP proteins anchor CAMP-dependent protein kinase to KvLQT1/IsK channel complex. Am J Physiol Heart Circ Physiol 2001, 280(5):H2038-H2045. 
10. Zaccolo M, Pozzan T: Discrete microdomains with high concentration of cAMP in stimulated rat neonatal cardiac myocytes. Science 2002, 295(5560):1711-1715.

11. Mauban JR, et al: AKAP-scaffolding proteins and regulation of cardiac physiology. Physiology (Bethesda) 2009, 24:78-87.

12. Jarnaess $E$, et al: Dual specificity A-kinase anchoring proteins (AKAPs) contain an additional binding region that enhances targeting of protein kinase A type I. J Biol Chem 2008, 283(48):33708-33718.

13. Miki K, Eddy EM: Single amino acids determine specificity of binding of protein kinase $A$ regulatory subunits by protein kinase $A$ anchoring proteins. J Biol Chem 1999, 274(41):29057-29062.

14. Gold MG, et al: Molecular basis of AKAP specificity for PKA regulatory subunits. Mol Cell 2006, 24(3):383-395.

15. Kinderman FS, et al: A dynamic mechanism for AKAP binding to RII isoforms of cAMP-dependent protein kinase. Mol Cell 2006, 24(3):397-408.

16. Colledge M, Scott JD: AKAPs: from structure to function. Trends Cell Biol 1999, 9(6):216-221.

17. Dodge K, Scott JD: AKAP79 and the evolution of the AKAP model. FEBS Lett 2000, 476(1-2):58-61.

18. Appert-Collin A, Baisamy L, Diviani D: Regulation of g protein-coupled receptor signaling by a-kinase anchoring proteins. J Recept Signal Transduct Res 2006, 26(5-6):631-646.

19. Dessauer CW: Adenylyl cyclase-A-kinase anchoring protein complexes: the next dimension in CAMP signaling. Mol Pharmacol 2009, 76(5):935-941.

20. Nijholt IM, et al: Neuronal AKAP150 coordinates PKA and Epac-mediated PKB/Akt phosphorylation. Cell Signal 2008, 20(10):1715-1724.

21. Baillie GS, Scott JD, Houslay MD: Compartmentalisation of phosphodiesterases and protein kinase A: opposites attract. FEBS Lett 2005, 579(15):3264-3270.

22. Bajpai M, et al: AKAP3 selectively binds PDE4A isoforms in bovine spermatozoa. Biol Reprod 2006, 74(1):109-118.

23. Dell'Acqua ML, et al: Regulation of neuronal PKA signaling through AKAP targeting dynamics. Eur J Cell Biol 2006, 85(7):627-633.

24. Moss SJ, et al: Functional modulation of GABAA receptors by CAMPdependent protein phosphorylation. Science 1992, 257(5070):661-665.

25. Hall DD, et al: Critical role of CAMP-dependent protein kinase anchoring to the L-type calcium channel Cav1.2 via A-kinase anchor protein 150 in neurons. Biochemistry 2007, 46(6):1635-1646.

26. Tunquist BJ, et al: Loss of AKAP150 perturbs distinct neuronal processes in mice. Proc Natl Acad Sci U S A 2008, 105(34):12557-12562.

27. Soderling SH, et al: Loss of WAVE-1 causes sensorimotor retardation and reduced learning and memory in mice. Proc Natl Acad Sci U S A 2003, 100(4):1723-1728

28. Schillace RV, Scott JD: Association of the type 1 protein phosphatase PP1 with the A-kinase anchoring protein AKAP220. Curr Biol 1999, 9(6):321-324

29. Henn $V$, et al: Identification of a novel A-kinase anchoring protein 18 isoform and evidence for its role in the vasopressin-induced aquaporin-2 shuttle in renal principal cells. J Biol Chem 2004, 279(25):26654-26665.

30. Robinson ML, et al: Association of the type I regulatory subunit of CAMPdependent protein kinase with cardiac myocyte sarcolemma. Arch Biochem Biophys 1996, 330(1):181-187.

31. Dodge-Kafka $K L$, et al: The protein kinase $A$ anchoring protein mAKAP coordinates two integrated cAMP effector pathways. Nature 2005, 437(7058):574-578

32. Kurokawa J, et al: Regulatory actions of the A-kinase anchoring protein Yotiao on a heart potassium channel downstream of PKA phosphorylation. Proc Natl Acad Sci U S A 2004, 101(46):16374-16378

33. Carnegie GK, et al: AKAP-Lbc mobilizes a cardiac hypertrophy signaling pathway. Mol Cell 2008, 32(2):169-179.

34. Russell MA, et al: The intermediate filament protein, synemin, is an AKAP in the heart. Arch Biochem Biophys 2006, 456(2):204-215.

35. Reynolds $J G$, et al: Identification and mapping of protein kinase $A$ binding sites in the costameric protein myospryn. Biochim Biophys Acta 2007, 1773(6):891-902.

36. Few WP, Scheuer T, Catterall WA: Dopamine modulation of neuronal $\mathrm{Na}$ $(+)$ channels requires binding of A kinase-anchoring protein 15 and PKA by a modified leucine zipper motif. Proc Natl Acad Sci U S A 2007, 104(12):5187-5192.

37. Cantrell AR, et al: Molecular mechanism of convergent regulation of brain $\mathrm{Na}(+)$ channels by protein kinase $\mathrm{C}$ and protein kinase $\mathrm{A}$ anchored to AKAP-15. Mol Cell Neurosci 2002, 21(1):63-80.
38. Lygren B, et al: AKAP complex regulates Ca2+ re-uptake into heart sarcoplasmic reticulum. EMBO Rep 2007, 8(11):1061-1067.

39. Hulme JT, et al: A novel leucine zipper targets AKAP15 and cyclic AMPdependent protein kinase to the $\mathrm{C}$ terminus of the skeletal muscle $\mathrm{Ca} 2+$ channel and modulates its function. J Biol Chem 2002, 277(6):4079-4087.

40. Hulme JT, et al: Beta-adrenergic regulation requires direct anchoring of PKA to cardiac CaV1.2 channels via a leucine zipper interaction with $A$ kinase-anchoring protein 15. Proc Natl Acad Sci U S A 2003, 100(22):13093-13098.

41. Bengrine A, Li J, Awayda MS: The A-kinase anchoring protein 15 regulates feedback inhibition of the epithelial $\mathrm{Na}+$ channel. FASEB J 2007 , 21(4):1189-1201.

42. Gray PC, Scott JD, Catterall WA: Regulation of ion channels by cAMPdependent protein kinase and A-kinase anchoring proteins. Curr Opin Neurobiol 1998, 8(3):330-334.

43. Dai S, Hall DD, Hell JW: Supramolecular assemblies and localized regulation of voltage-gated ion channels. Physiol Rev 2009, 89(2):411-452.

44. Hundsrucker C, et al: High-affinity AKAP7delta-protein kinase A interaction yields novel protein kinase A-anchoring disruptor peptides. Biochem J 2006, 396(2):297-306.

45. Christian F, et al: Small molecule AKAP-protein kinase A (PKA) interaction disruptors that activate PKA interfere with compartmentalized CAMP signaling in cardiac myocytes. J Biol Chem 2011, 286(11):9079-9096.

46. Trotter KW, et al: Alternative splicing regulates the subcellular localization of A-kinase anchoring protein 18 isoforms. J Cell Biol 1999, 147(7):1481-1492.

47. Brown RL, et al: AKAP7gamma is a nuclear Rl-binding AKAP. Biochem Biophys Res Commun 2003, 306(2):394-401.

48. Hausken ZE, et al: Type II regulatory subunit (RII) of the cAMP-dependent protein kinase interaction with A-kinase anchor proteins requires isoleucines 3 and 5. J Biol Chem 1994, 269(39):24245-24251.

49. Li Y, Rubin CS: Mutagenesis of the regulatory subunit (RII beta) of CAMPdependent protein kinase II beta reveals hydrophobic amino acids that are essential for RII beta dimerization and/or anchoring RII beta to the cytoskeleton. J Biol Chem 1995, 270(4):1935-1944.

50. Lygren B, Tasken KA: The potential use of AKAP18delta as a drug target in heart failure patients. Expert Opin Biol Ther 2008, 8:1099-1108.

51. Tröger J, Moutty MC, Skroblin P, Klussmann E: A-kinase anchoring proteins as potential drug targets. Br J Pharmacol 2012, 166(2):420-33. doi:10.1111/j.1476-5381.2011.01796.x

52. Canaves JM, Taylor SS: Classification and phylogenetic analysis of the CAMP-dependent protein kinase regulatory subunit family. J Mol Evol 2002, 54(1):17-29.

53. Singh A, et al: The large isoforms of A-kinase anchoring protein 18 mediate the phosphorylation of inhibitor- 1 by protein kinase $A$ and the inhibition of protein phosphatase 1 activity. Mol Pharmacol 2011, 79(3):533-40.

54. Josefsen $\mathrm{K}$, et al: AKAP 18 alpha and gamma have opposing effects on insulin release in INS-1E cells. FEBS Lett 2010, 1:81-5.

55. Gray PC, et al: Identification of a $15-k D a$ CAMP-dependent protein kinaseanchoring protein associated with skeletal muscle L-type calcium channels. J Biol Chem 1997, 272(10):6297-302.

56. Gray $P C$, et al: Primary structure and function of an A kinase anchoring protein associated with calcium channels. Neuron 1998, 20(5):1017-26.

57. Fu Y, et al: Deletion of the distal $C$ terminus of CaV1.2 channels leads to loss of beta-adrenergic regulation and heart failure in vivo. $J \mathrm{Biol}$ Chem. 2011, 286(14):12617-26.

58. Finn RD, et al: The Pfam protein families database. Nucleic Acids Res 2008 38(Database issue):D211-22.

59. Tamura $K$, et al: MEGA5: molecular evolutionary genetics analysis using maximum likelihood, evolutionary distance, and maximum parsimony methods. Mol Biol Evol 2011, 28(10):2731-9.

60. Burge C, Karlin S: Prediction of complete gene structures in human genomic DNA. J Mol Biol 1997, 268(1):78-94.

61. Knudsen S: Promoter2.0: for the recognition of Polll promoter sequences. Bioinformatics 1999, 15(5):356-61.

doi:10.1186/1471-2148-12-125

Cite this article as: Johnson et al:: Molecular evolution of a-kinase anchoring protein (AKAP)-7: implications in comparative PKA compartmentalization. BMC Evolutionary Biology 2012 12:125. 